PROPUESTA DE UN MATERIAL DIDÁCTICO PARA LA ENSEÑANZA APRENDIZAJE DE POLINOMIOS PARA POBLACIÓN CON LIMITACIÓN VISUAL

\title{
Propuesta de un material didáctico para la enseñanza aprendizaje de polinomios para población con limitación visual ${ }^{1}$
}

\author{
Proposal for teaching materials for the learning of \\ polynomials for visualla population limitation
}

Proposta de materiais para a aprendizagem de polinômios para a limitação da população visualla ensinando.

Recibido: mayo de 2013

Aceptado: agosto de 2013
Ramiro Adolfo Jiménez Leal ${ }^{2}$ Dumar Barreto Perilla ${ }^{3}$

Fabio Fernando Funeme Rosero ${ }^{4}$

\section{Resumen}

En el presente artículo se dará a conocer una propuesta de un material didáctico que permita a estudiantes invidente, trabajar el concepto de polinomios dentro de un aula regular, esto con el propósito de abordar las necesidades que tienen los estudiantes invidentes en el aula regular, cuando trabaja con el área de matemáticas, específicamente con el álgebra.

Palabras clave: Material didáctico; Alumno; Necesidades especiales; Estudiantes discapacitados; Estudiantes invidentes; Matemáticas escolares; Álgebra; Polinomios; Álgebra geométrica; Aula regular.

\begin{abstract}
In this article we will present a proposal for a teaching aid that allows blind students, working with the concept of polynomials in a regular classroom, this in order to address the needs that blind students in the regular classroom, when works in the area of mathematics, specifically algebra.

Keywords: Textbooks, Student, Special Needs, Students with Disabilities, Students blind; school Math, Algebra, Polynomials, Algebra Geometrical Regular Classroom.
\end{abstract}

\section{Resumo}

Neste artigo, vamos apresentar uma proposta de um auxiliar de ensino que permite que os alunos cegos, trabalhando com o conceito de polinômios em uma sala de aula regular, isto a fim de atender às necessidades que os alunos cegos na sala de aula regular, quando trabalhos na área da matemática, especificamente álgebra.

1 Artículo de Investigación.

2 Universidad distrital francisco José de Caldas, Bogotá, Colombia. Contacto: ramir3399@hotmail.com

3 Universidad distrital francisco José de Caldas, Bogotá, Colombia. Contacto: dubareto1982@hotmail.com

4 Universidad distrital francisco José de Caldas, Bogotá, Colombia. Contacto: fabiofuneme@yahoo.com 
Palavras-chave: Livros didáticos, estudantes, necessidades especiais, alunos com deficiência, alunos cegos; matemática escolar, álgebra, polinômios, Álgebra Geométrica aula regular.

\section{Contextualización}

Dentro de la enseñanza de las matemáticas en un aula inclusiva, el docente debe llevar a cabo una ardua labor de documentación, valiéndose de referentes teóricos que le permitan abordar con mayor responsabilidad las necesidades que pueda presentar un estudiante con o sin discapacidad, también "frente a los contenidos curriculares en la interacción con su contexto escolar y que, para satisfacerlas, requiere de apoyo educativo de carácter adicional o diferente" (Espejo, 2001).

En esta medida, debido a que hacemos parte del Proyecto Curricular de Licenciatura En Educación Básica Con Énfasis En Matemáticas, de la Universidad Distrital; en ella se establece como requisito para que el estudiante se gradúe, la culminación de los espacios de formación y la elaboración del trabajo de grado. Para el segundo requisito, una de las modalidades que presenta la universidad como trabajo de grado, y la cual se selecciono para realizar, es la modalidad de pasantía de extensión orientada a las necesidades educativas especiales (NEES). En esta modalidad, se trabajo específicamente con estudiantes invidentes del colegio José Félix Restrepo, de la localidad Antonio Nariño. Dentro de la institución se debía cumplir con tres procesos, los cuales eran acompañamiento en el aula, apoyo extra clase y adaptación de material.

Durante este proceso se trabajo con estudiantes invidentes entre los 25 y 40 años del grado noveno, de la jornada nocturna. En el proceso de enseñanza aprendizaje se evidenció una problemática con respecto a la falta de material didáctico adaptado que permitiera afrontar conceptos matemáticos, específicamente en el álgebra, no solo en la institución educativa si no a nivel general, por esto se inicio un proceso de investigación que permitiera dar cuenta del como diseñar un material adecuado para un estudiante invidente, que le permita tanto desenvolverse dentro del aula regular como acercarse a los conceptos matemáticos-algebraicos.

Teniendo en cuenta lo dicho anterior mente, en este trabajo se presenta una propuesta de material didáctico que permite trabajar con estudiantes invidentes el concepto algebraico de polinomios (además que este mismo permite potenciar otros conceptos matemáticos).

\section{Marco teórico - Prácticos básicos}

Para la elaboración del material didáctico, se inicio un análisis desde el algebra geométrica como recurso didáctico para la adquisición y comprensión de la noción de polinomios. En esta medida, por un lado haciendo referencia a Socas M. (1996), menciona que los griegos para la resolución de ecuaciones desarrollaron métodos geométricos; por ejemplo, en el libro II de los elementos de Euclides (300 a. de C.), hay 14 proposiciones que permiten resolver problemas algebraicos, y ecuaciones cuadráticas con un procedimiento llamado "aplicación de áreas". Y por otro lado haciendo alusión a lo dicho por el grupo Azarquiel (1993), propone que para poder comprender el sentido de los símbolos es necesario interiorizar la doble relación entre las situaciones concretas y las expresiones algebraicas.

Cabe agregar que en el algebra, la representación simbólica y la geométrica van de la mano en el proceso de enseñanza aprendizaje, ya que permiten una mayor comprensión en el estudiante, desde la perspectiva de Radford (1991) el simbolismo y la geometría tienen un papel muy importante; el símbolo por tener la capacidad de representar lo desconocido con un carácter de variabilidad en donde se forma un lenguaje discursivo propio del álgebra; la 
geometría se convierte en una herramienta manipulativa, intuitiva, deductiva y analítica en la que pueden existir magnitudes desconocidas que se pueden llegar a asociar con el lenguaje discursivo.

Teniendo en cuenta las ideas expuestas anteriormente, se observa que el algebra geométrica permite realizar adaptaciones de material tanto para el estudiante con o sin discapacidad visual. Ahora, Para comprender mejor lo de material didáctico para personas con discapacidad visual citamos a Rosich (1996), quien intenta distinguir algunas etapas que se producen en la cognición matemática a bajo nivel, no sólo para esta población en particular, de estas, solo citaremos las dos iniciales:

a. Recogida de información sensible: Un contenido matemático puede presentarse bajo diferentes ropajes sensibles como lo es el visual, audible, háptico ${ }^{5}$; capaz de estimular los correspondientes receptores sensoriales (p. 157). Para la población ciega, esta primera fase demanda una serie de destrezas que deberán haber sido adquiridas de antemano.

b. Elaboración del correspondiente percepto ${ }^{6}$ : esta fase tiene que ver con la forma como el sujeto elabora una representación del objeto matemático a partir de su percepción mediante los canales referenciados en la fase anterior.

\section{Descripción}

Este trabajo se implemento en el colegio I.E.D. JOSE FELIX RESTREPO, a estudiantes de un aula inclusiva (con estudiantes invidentes) del grado noveno, de la jornada nocturna. Inicialmente en el proceso de apoyo extra clase, se trabajo con los estudiantes invidentes (2) las operaciones de suma y producto con monomios, binomios y trinomios, pero debido a que los estudiantes presentaban dificultad al realizar las operaciones debido a la posible extensión de términos, se busco una herramienta que le fuese más fácil operarlos, y que le permitiera ir reconociendo el resultado obtenido. Esta herramienta fue realizada con materiales que permitieran a los estudiantes invidentes hacer uso del sistema haptil, como es la escritura braille (en las fichas) y manejo de diferentes texturas en el tablero; a continuación presentamos la forma del material:

Figura 1

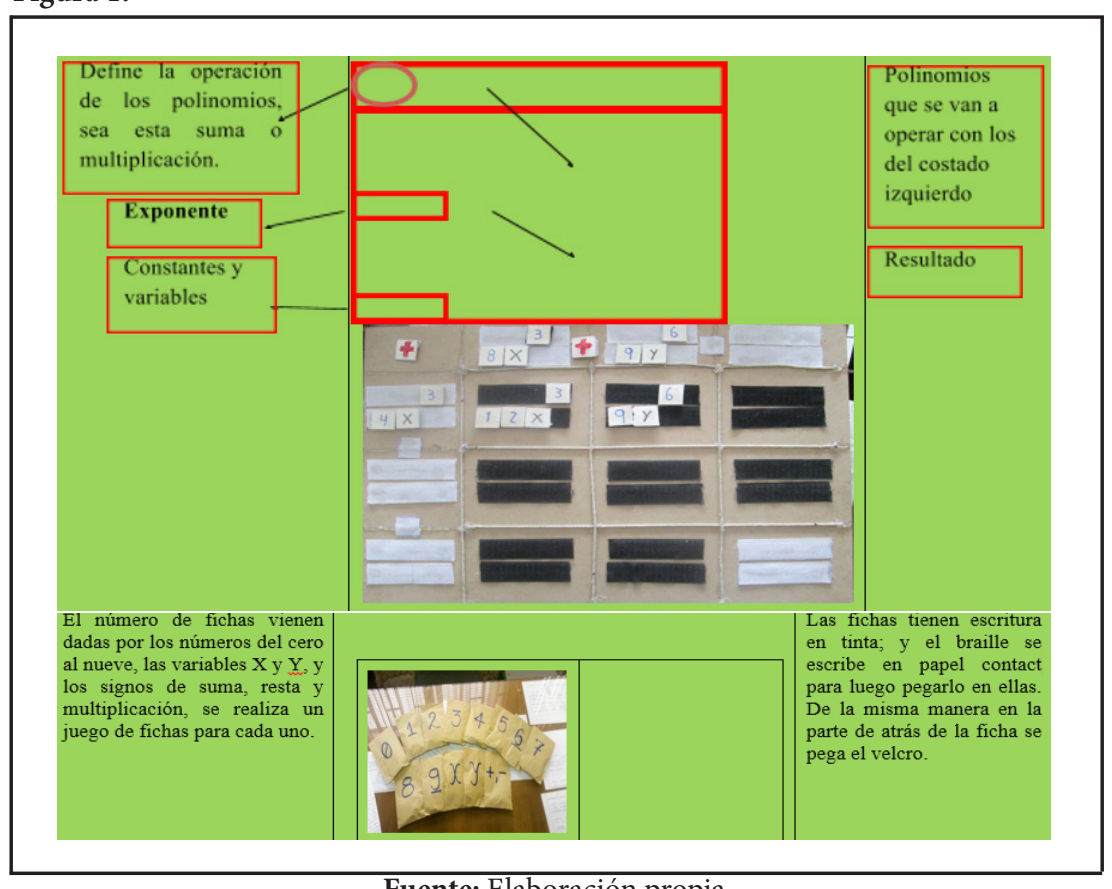

Fuente: Elaboración propia

5 Referente al tacto.

6 Se refiere al objeto tal como lo percibe el sujeto 
Con las herramientas diseñadas por el grupo de trabajo cuyo principal objetivo es realizar un material inclusivo, que permita al estudiante con discapacidad visual acceder al conocimiento matemático y a la participación en el aula de clase; se procedió de la siguiente manera:

El material se implemento inicialmente en el proceso de apoyo extra clase, en donde se realizan refuerzos personales a los estudiantes invidentes con relación a las temáticas que abordan dentro del aula de matemáticas, aquí se les presento el material a los estudiantes (2) para que reconocieran su formas y texturas del material, luego se le describió que función realizaba cada uno de los belcros puestos y como deberían ir ubicadas las fichas dadas en los mismos. Reconociendo el material se procedió a realizar una serie de ejercicios que el estudiante debía realizar de la clase de matemáticas, iniciándose con monomios, binomios y trinomios, observándose buenos resultados por el desenvolvimiento del estudiante con el instrumento en la solución de los mismos, además que uno de los estudiantes afirmaba que esto le permitía reconocer que dada una expresión con cual debía esta realizarse la operación.

Observando los buenos resultados al hacer uso del material, se hace uso del mismo dentro del aula de clase. Este proceso se conoce como acompañamiento en el aula, en donde el pasante es solo una ayuda para el estudiante invidente. En el transcurso de la clase el docente titular pide a los estudiantes realizar la solución de uno de los polinomios, de modo que los dos estudiantes pasan al frente con el instrumento, y exponen satisfactoriamente la solución del polinomio.

\section{Logros}

- la adaptación del material permitió un mayor acercamiento al concepto de polinomios a los estudiantes invidentes, generando dudas y aclaraciones sobre el mismo.

- El material permitió una participación activa del estudiante dentro del aula, de tal modo que realizaron exposiciones frente a sus compañeros de clase.

\section{Dificultades}

- Desconocimiento por parte de uno de los estudiantes del uso de las variables puestas en juego dentro de un polinomio.

- Falta de comprensión de los conceptos y propiedades que involucran a los polinomios que dirige a que el significado de las operaciones y relaciones se asocien a procesos algorítmicos.

\section{Reflexión final}

Con las herramientas diseñadas por el grupo de trabajo se pudo alcanzar dos objetivos muy importantes, en lo que se pretende en una aula inclusiva, estos son: que los estudiantes invidentes comprendan los conceptos matemáticas (polinomios) y, que fuesen participantes activos dentro del aula de clase, al realizar exposiciones y participar en los grupos de trabajo, etc.

En el proceso de apoyo extra clase se abordaron los errores conceptuales que los estudiantes presentaban al realizar la adición y producto entre las variables. (kieran $1981 \mathrm{Al}$ usar la letra como variable tiene sentido el producto $2 x{ }^{*} 3 y=6 x y$, lo cual no funciona de igual manera para la suma: $2 x+3 y=\ldots$

El trabajo en un aula inclusiva, en donde hallan estudiantes con discapacidad visual, se puede transformar en un ambiente equitativo con respecto al acceso al conocimiento matemático (y cualquier 
otra área del conocimiento) ya que las matemáticas además de abordar una gran extensión de conceptos, también permite que estos se materialicen y sean accesibles a todo tipo de población.

\section{Referencias}

Espejo, J. (2001). Antecedentes, marco legal y psicopedagógico de la educación especial en México. En Pedagogía y diversidad. Convenio Andrés Bello. La Habana. Casa editora Abril. P 29- 43 .

Montero, J. (2002). Enseñar matemáticas a alumnos con necesidades educativas espaciales. Barcelona. Editorial Praxis.

Radford, L. (1991). The roles of geometry and aritmetic in the development of algebra: historical remarks from a didactic perspective.

Rojas, P. Y otros (1999) Transición aritmética-algebra: actividades para potenciar diversas interpretaciones de la letra. universidad distrital francisco José de caldas

Rosich, N. (1998) Matemáticas y deficiencia sensorial. Madrid. Editorial síntesis

Socas, M. y otros (1989). Iniciación al álgebra. Madrid-España.

Stainback, S y Stainback, W. (1999). Aulas inclusivas: Un nuevo modo de enfocar y vivir el currículo. Madrid. Narcea, S.A de ediciones. 